\title{
Erythrocyte Senescent Markers by Flow Cytometry
}

\section{María Alejandra Ensinck, Melina Eliana Luján Brajovich, Silvia Estela García Borrás, Carlos Miguel Cotorruelo, Claudia Silvia Biondi}

\author{
Department of Clinical Biochemestry, Faculty of Biochemical and Pharmaceutical Sciences, National University of Rosario, \\ Rosario, Argentina \\ Email: mensinck@fbioyf.unr.edu.ar, mlujan@fbioyf.unr.edu.ar, sigarcia@fbioyf.unr.edu.ar, ccotorru@fbioyf.unr.edu.ar, \\ cbiondi@fbioyf.unr.edu.ar
}

How to cite this paper: Ensinck, M.A., Brajovich, M.E.L., Borrás, S.E.G., Cotorruelo, C.M. and Biondi, C.S. (2019) Erythrocyte Senescent Markers by Flow Cytometry. Open Journal of Blood Diseases, 9, 47-59. https://doi.org/10.4236/ojbd.2019.93006

Received: August 9, 2019

Accepted: September 14, 2019

Published: September 17, 2019

Copyright $\odot 2019$ by author(s) and Scientific Research Publishing Inc. This work is licensed under the Creative Commons Attribution International License (CC BY 4.0).

http://creativecommons.org/licenses/by/4.0/

\begin{abstract}
Background: Mature red blood cells lack protein synthesis and are unable to restore inactivated enzymes, damaged cytoskeleton and membrane proteins. An oxidation breakdown of band 3 is probably part of the mechanism leading to the generation of a senescent cell antigen. This specific signal serves for the clearance of RBCs by inducing the binding of autologous IgG and C3, leading to phagocytosis. In addition, phosphatidilserin molecules appear in the outer membrane and the CD47 expression diminishes. Methods: Erythrocytes of different ages from whole blood were studied by flow cytometry analysing light scatter proprieties, binding of autologous IgG, C3 complement deposits, externalization of phosphatidylserine and CD47 expression. Dot-plot analysis based on forward scatter versus side scatter parameters showed two RBCs populations of different sizes and density. RBCs were further incubated with Alexa 488 IgG, APC-anti-C3, PE-annexin-V and PE-CD47. The comparison of the values obtained for the different variables studied in SeRBC and YRBC populations was carried out by the Student $t$-test for matched samples or by the Wilcoxon test (after verification of the normality assumption). Results: The percentage of IgG and C3 positive cells was significantly higher in senescent red blood cells population. The fraction of annexin-V positive RBCs was also larger in SeRBCs while the CD47 expression was lower in this population. Conclusions: These results indicate that flow cytometry allow differenciation of erythrocytes populations of different ages, turning this tool into an useful alternative option to study erythrocyte aging process. These findings will contribute to a better understanding of the process and mechanisms involved in erythrocyte senescence process.
\end{abstract}

\section{Keywords}

Erythrocyte, Senescence, Flow Cytometry 


\section{Introduction}

In healthy individuals, the number of erythrocytes exceeds $4 \times 10^{12} / \mathrm{L}$ in blood stream and has a mean lifespan of 115 days with a variation between 70 and 140 days, and a difference of $15 \%$ between individuals. Erythrocyte life is limited by senescence and subsequent removal of aged red blood cells (RBCs) [1] [2] [3].

Under physiological conditions, all individuals have physiologic autologous IgG antibodies to band 3 that bind to senescent (Se) RBCs and initiate their removal by macrophage. The accumulation of this specific antibody at the end of erythrocyte life represents an accessible evidence to identify the antigen and subsequently the aging mechanism [4] [5]. The small amount of autologous IgG found in vivo indicates that removal of SeRBCs is an efficient and controlled process. Naturally occurring antibodies (NAbs) that bind to band 3 are implicated in the clearance of senescent RBCs. These antibodies have the capacity to stimulate C3b deposition indicating that an effective phagocytosis requires an active complement system, implying its participation in SeRBC clearance [6] [7].

Exposure of phosphatidilserin (PS) in the outer layer membrane has been described as a marker of apoptosis in nucleated cells [8] [9]. Although apoptosis in RBCs remains controversial, erythrocytes show signs of PS exposure and membrane vesicles formation and release [10]. A decrease in phospholipid asymmetry might be one of the physiological aging consequences at membrane level, and in conjunction with other signals, contributes to senescent erythrocytes recognition and removal by phagocytes [11] [12].

The exact nature of the "eat me" signals on RBCs membrane remains undiscovered. It has been generally accepted that CD47 is a "don't eat me" signal that plays a crucial role in RBCs homeostasis. CD47 is a ubiquitously expressed protein that binds to the inhibitory signal-regulatory protein alpha receptor (SIRP $\alpha$ ) present on macrophages and other myeloid cells. The CD47-SIRP $\alpha$ interaction inhibits immune responses such as phagocytosis. Besides functioning as a "don't eat me" signal, some investigators have shown that a conformational change in the CD47 protein can switch the molecule from a "don't eat me" to an "eat me" signal. This change in the structure of CD47 can be induced by oxidative stress and promotes trombospondin-1 (TSP-1) binding to CD47, which creates a new binding site for SIRP $\alpha$. This alternative binding site for SIRP $\alpha$ induces a pro/phagocytic signal for the macrophage. The dual role of CD47-SIRP $\alpha$ in regulating RBCs uptake demonstrates the RBCs clearance complexity [13] [14] [15] [16].

There is evidence that RBCs tend to become denser as they age, and many studies have used density as a surrogate marker. Nevertheless, it has been a matter of some controversy whether the enrichment of older RBCs in the dense fraction is adequate for this purpose. The increased density is not uniformly associated with age, and the dense fraction might contain a selected population of old cells that may not be representative of the majority. The complex temporal sequence invalidates the use of density as an unique parameter to study RBCs aging [7] [17]. 
Flow Cytometry (FC) is a very useful tool for cell analysis since it allows the objectively individual study of a large number of cells with high sensitivity and specificity.

The aim of this work was to evaluate IgG, C3 complement, PS and CD47 expression levels on RBCs from different ages by flow cytometry.

\section{Materials and Methods}

Blood samples $(\mathrm{n}=38)$ were collected from healthy donors into EDTA tubes and stored at $4^{\circ} \mathrm{C}$ for less than $24 \mathrm{~h}$ until analyzed. 20 of the donors were female and 18 were male. The average age was between 24 and 43 years old.

The study protocol was accepted by the ethical committee of the School of Biochemistry Sciences of Rosario, Argentina and all participants provided written consent according to the principles of the Declaration of Helsinki. The date of approvement by the ethics committee was February 21, 2019 by means of decanal resolution No. 065/2019.

\subsection{Flow Cytometric Analyses}

Flow cytometric analyses were performed on a FACSAria II flow cytometer (Becton Dickinson, San Jose, CA, USA). The FACSDiva software was used for acquisition and analysis. The light-scatter and the fluorescence channels were set on a logarithmic scale, a minimum of 100,000 cells were analyzed in each condition.

\subsection{Autologous IgG on RBCs Evaluation}

The IgG bound to the erythrocytes membrane surface was analysed in fractions of RBCs from different age separated by Percoll gradients and in populations obtained according to the information of light scattering parameters (FSC and SSC) analysed by FC.

The erythrocyte suspensions evaluated were:

- Suspension 1: SeRBCs and young (Y) RBCs populations obtained according to Percoll separation [18].

- Suspension 2: RBCs from whole blood samples.

After 4 washes with $3 \mathrm{ml}$ of phosphate-buffered saline solution (PBS; pH 7.4; $137 \mathrm{mM} \mathrm{NaCl}, 2.7 \mathrm{mM} \mathrm{KCl}, 8.1 \mathrm{mM} \mathrm{Na} \mathrm{HPO}_{4}$, and $1.5 \mathrm{mM} \mathrm{KH}_{2} \mathrm{PO}_{4}$ ), suspensions were prepared to concentration of $10^{6}$ cells $/ \mathrm{mL}$ PBS from each RBCs populations. $50 \mu \mathrm{L}$ of each suspension were incubated with $50 \mu \mathrm{L}$ of anti-human IgG labeled with Alexa Fluor 488 (Molecular Probes, Invitrogen, USA) diluted 1:100. After 60 minutes of incubation in the dark, the suspensions were washed once with PBS and resuspended in $0.5 \mathrm{ml}$ of FACS Flow diluent (BD Biosciences) [17].

The following controls were used:

Positive control: RBCs suspensions sensibilizated with IgG.

Negative control: Unlabeled RBCs suspensions. 
The statistical analysis by Wilcoxon test for paired samples showed that the percentage of IgG positive (IgG+) cells do not differ significantly between the populations obtained by both methodologies [18]. The comparable results with the samples without previous separation allowed the study of other senescence markers using whole blood without any separation step, evaluating each marker in the regions defined by FSC and SSC light scattering parameters.

\subsection{C3 Complement Analysis}

The evaluation of $\mathrm{C} 3$ bound to erythrocytes membrane surface was carried out by FC in whole blood samples. RBCs were washed and diluted to a concentration of $10^{6}$ cells $/ \mathrm{mL}$ in PBS. $50 \mu \mathrm{L}$ of RBCs suspension were incubated with $10 \mu \mathrm{L}$ of anti-C3b (Bioclone, Ortho Diagnostic Systems). After 30 minutes of incubation, a wash with PBS was performed. $50 \mu \mathrm{L}$ of the allophicocyanin-labeled anti-mouse secondary antibody (APC) (BD Pharmingen USA) was added and incubated 30 min in the dark. The RBCs were washed once with PBS and resuspended in 0.5 $\mathrm{ml}$ of FACS Flow diluent (BD Biosciences).

\subsection{Flow Cytometric Analyses of Phosphatidylserine Exposure}

The evaluation of PS expression on the RBCs external surface is based on its specific binding to annexin V (Annexin V Apotosis Detection Kit I.BD Pharmingen USA). Suspensions were prepared to a concentration of $10^{6}$ cells $/ \mathrm{mL}$ in buffer with calcium (Annexin Binding Buffer). $25 \mu \mathrm{L}$ of each suspension were incubated with $5 \mu \mathrm{L}$ of annexin V-phycoerythrin labelled (PE) for 30 minutes in the dark. Subsequently, the samples were washed once with calcium buffer and $\mathrm{RBCs}$ were resuspended in $0.5 \mathrm{~mL}$ of FACSFlow diluent (BD Biosciences).

Negative control: unlabeled RBCs resuspended in calcium buffer.

Positive control: the permeabilization of the erythrocytes was carried out by incubating $50 \mu \mathrm{L}$ of washed RBCs with $50 \mu \mathrm{L}$ of $50 \%$ ethanol in PBS, 2 minutes at $39^{\circ} \mathrm{C}$. The RBCs were washed and resuspended in Annexin Binding Buffer. 25 $\mu \mathrm{L}$ of this suspension was incubated with $5 \mu \mathrm{L}$ of annexin V-PE for 30 minutes in the dark. Subsequently, the RBCs were washed with calcium buffer and resuspended in $0.5 \mathrm{~mL}$ of FACSFlow diluent (BD Biosciences). Under these conditions, annexin $\mathrm{V}$ binds to the exposed PS in the outer layer membrane as well as to the PS present in the inner membrane.

\subsection{Assessment of CD47 Expression on RBCs}

The surface expression of CD47 on RBCs was assessed using a monoclonal antibody anti-CD47 conjugated with PE (Clone B6H12. BD Pharmingen USA). For this purpose, $50 \mu \mathrm{L}$ of washed RBCs at a concentration of $10^{6}$ cells $/ \mathrm{mL}$ in PBS were incubated with $5 \mu \mathrm{L}$ of anti-CD47 antibody in darkness at room temperature for $15 \mathrm{~min}$. After staining, cells were washed once with PBS and resuspended in $0.5 \mathrm{ml}$ FACSFlow solution (Becton Dickinson). Median fluorescence intensity (MFI) served as the measure of expression level and was compared between both populations. 


\subsection{Statistical Analysis}

To verify compliance with the assumption of normality required by the different statistical techniques used the Shapiro-Wilk test was applied [18].

The statistical comparison of the results obtained for the different parameters analyzed in SeRBC populations separated by Percoll preformed gradients and studied in whole blood, was carried out using the Wilcoxon test.

The comparison of the values obtained for the different variables studied in SeRBC and YRBC populations was carried out by the Student $t$-test for matched samples or by the Wilcoxon test (after verification of the normality assumption).

\section{Results}

\subsection{Autologous IgG Evaluation in RBCs}

Based on data from Kay [19] [20] [21], showing that autologous IgG plays a role in the selective clearance of senescent RBCs, we searched for naturally occurring IgG antibodies (NAbs) directed against RBC proteins. The average values of IgG+ cells percentages in different RBCs suspensions are shown in Table 1. In SeRBCs population, the average values of IgG+ cells percentages were significantly higher than those observed in YRBCs $(\mathrm{p}<0.002)$ when studying erythrocyte populations obtained by both methods. The statistical analysis showed that the values of IgG+ cells percentages in SeRBCs and YRBCs populations do not differ significantly between the methodologies used in this work. The comparable results in samples without a previous separation step, allowed us to continue the subsequent study of other RBCs senescence markers analysing whole blood, evaluating each marker in regions defined by FSC and SSC light scattering parameters (Figure 1). The analysis strategy used and the results of IgG+ percentage obtained in suspensions 1 and 2 are shown in Figure 2.
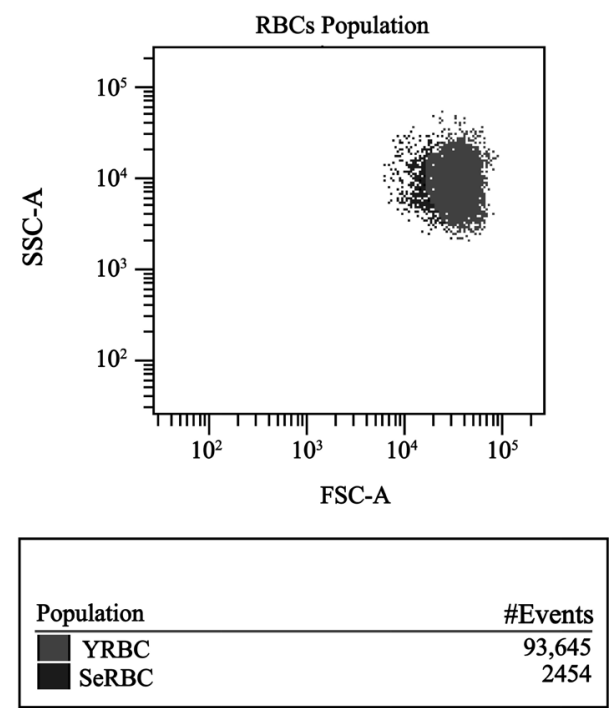

Figure 1. Dot-plot of whole blood with the analysis strategy used to evaluate RBCs from different ages. 

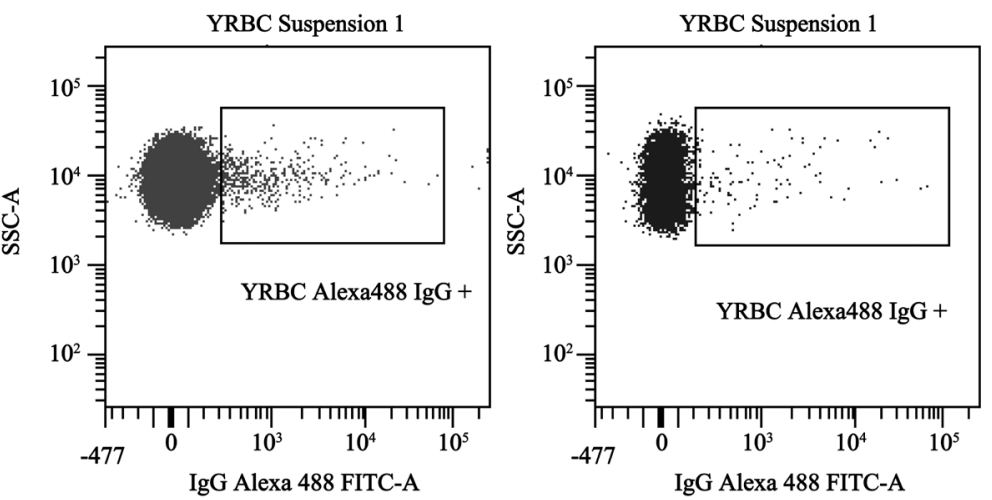

\begin{tabular}{|lrr|}
\hline \multicolumn{2}{|l|}{ Specimen Name: Suspension 1 YRBC } & \\
Population & \#Events & \% Parent \\
\hline$\square$ RBC & 95,679 & 95.7 \\
YRBC Alexa488 IgG+ & 536 & 0.6 \\
SeRBC Alexa488 IgG+ & 119 & 3.1 \\
\hline
\end{tabular}
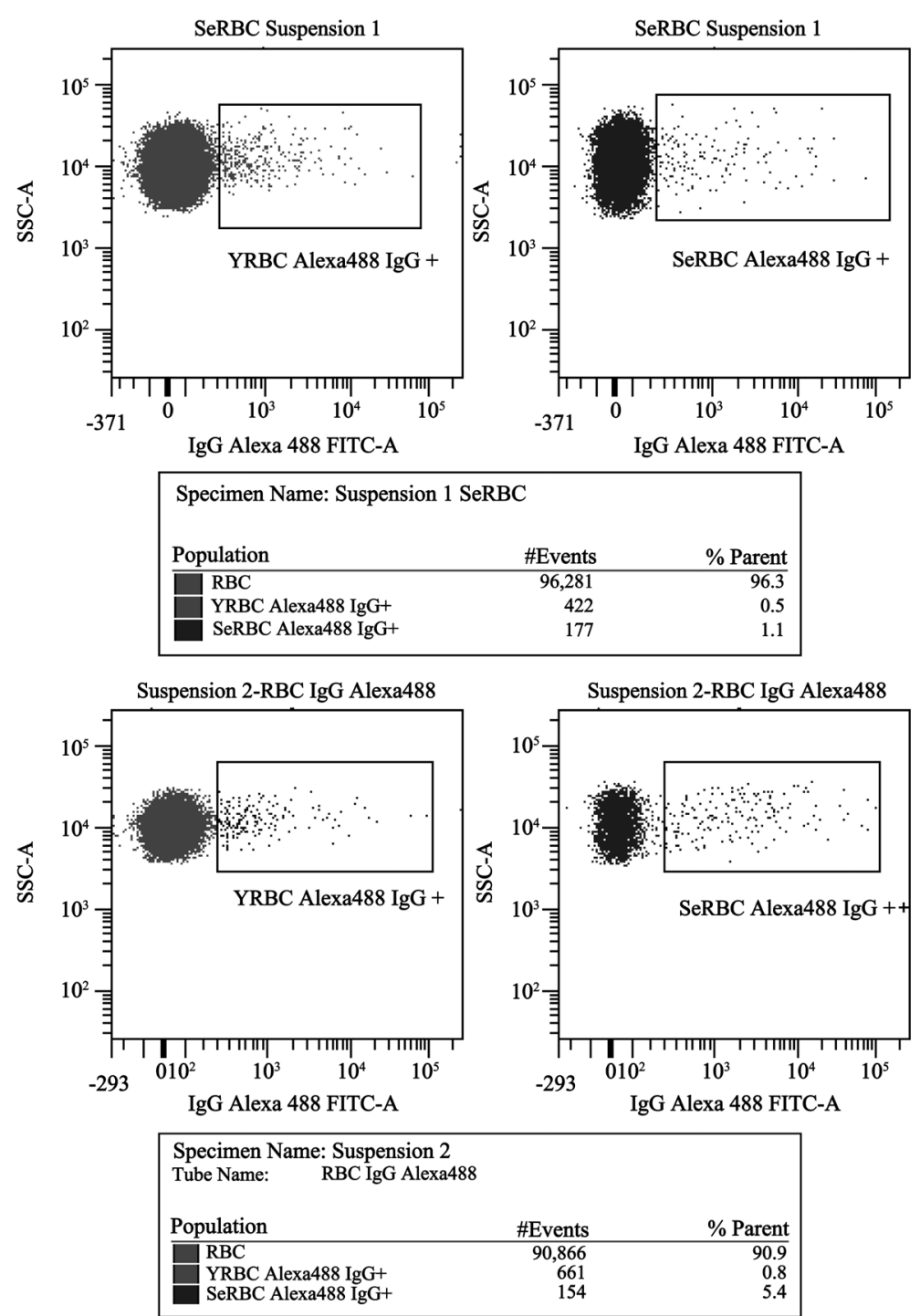

Figure 2. IgG+ RBCs in populations separated by Percoll gradients and populations analysed by light scattering parameters (FSC and SSC). 
Table 1. IgG+ cells \% (mean \pm standard deviation). Suspension 1: RBCs separated by Percoll gradients. Suspension 2: Whole blood.

\begin{tabular}{llc}
\hline & \multicolumn{2}{c}{$\%$ of RBCs IgG+ } \\
\hline & SeRBCs & YRBCs \\
\hline Suspension 1 & $2.6 \pm 1.3$ & $0.4 \pm 0.3$ \\
Suspension 2 & $2.3 \pm 1.4$ & $0.4 \pm 0.3$ \\
\hline
\end{tabular}

\subsection{C3 Complement Analysis}

Naturally occurring auto-antibodies activate the classical complement pathway and stimulate complement amplification and C3b component deposition. Therefore, complement amplification compensates the low affinity of NAbs and generates efficient opsonins. Table 2 describes the median values of $\mathrm{C} 3+\mathrm{RBCs}$ percentages of erythrocytes without physical separation. Data corresponding to SeRBCs population was significantly higher than the values obtained from YRBCs $(\mathrm{p}<0.0001)$ (Figure 3$)$.

\subsection{Phosphatidylserine Externalization Measurement}

We also assessed phosphatidylserine exposure on the outer leaflet plasma membrane, which acts as a signal triggering erythrophagocytosis. We observed difference in the phosphatidylserine externalization level between SeRBCs and YRBCs (Figure 4). Table 3 shows the percentages of RBCs with increased PS on the outer side membrane, based on annexin $\mathrm{V}$ binding affinity. The median value of annexin $\mathrm{V}$ positive cells $(\mathrm{AV}+)$ percentage was significantly higher in SeRBC compared to YRBCs $(\mathrm{p}<0.001)$.

\subsection{Assessment of CD47 Expression on RBCs}

Recent studies have demonstrated that CD47 membrane antigen is an important self-recognition marker involved in protecting the circulating RBCs from phagocytosis [13]. Comparative analyses of membrane CD47 expression in SeRBCs versus YRBCs showed a general decrease in MFI values in senescent erythrocytes population (Figure 5). The median of CD47 MFI was significantly lower in SeRBCs than in YRBCs $(\mathrm{p}<0.001)$, as shown in Table 4.

\section{Discussion}

Erythrocyte senescence is associated with cell shrinkage, plasma membrane vesiculization, progressive shape change from discocytes to spherocytes, cytoskeletal and membrane proteins alteration. This process is also characterized by changes in the plasma membrane phospholipids asymmetry that lead to the externalization of PS, which may represent one of the signals that would allow SeRBCs macrophages ingestion [22] [23] [24].

The major problem in studying SeRBCs properties is the lack of a reliable method to isolate RBCs populations. Density gradient separation methods have allowed the collection of the densest human RBCs that would be destined for 
immediate clearance [2] [12] [25] [26]. However, separation techniques used are laborious and require a considerable amount of sample.
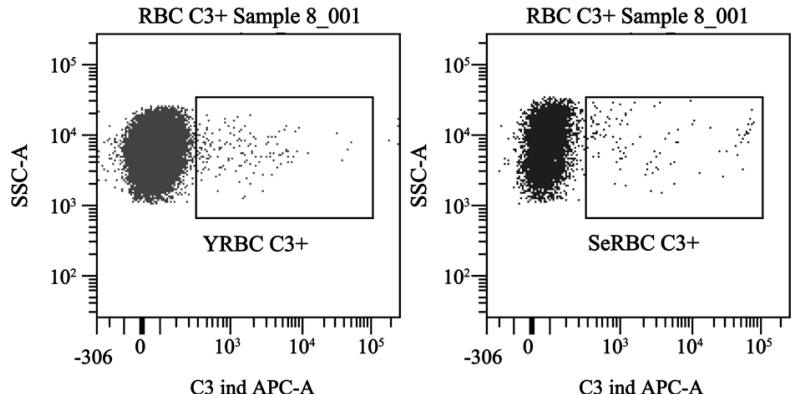

\begin{tabular}{|lrr|}
\hline Specimen Name: RBC C3+ & & \\
& & \\
Population & \#Events & \% Parent \\
\hline RBC & 93,853 & 93.9 \\
YRBC C3+ & 307 & 0.3 \\
SeRBC C3+ & 113 & 1.9 \\
\hline
\end{tabular}

Figure 3. Dot-plot of whole blood with the analysis strategy used to evaluate C3+ RBCs in populations from different ages.
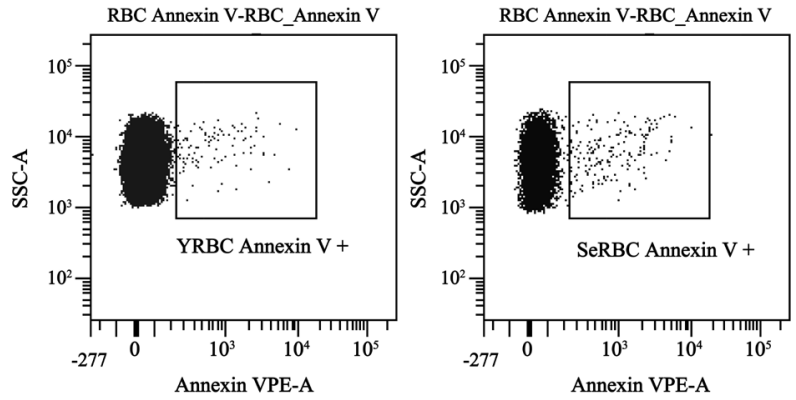

\begin{tabular}{|lrr|}
\hline Specimen Name: RBC Annexin V & & \\
& & \\
Population & \#Events & \% Parent \\
\hline RBC & 85,636 & 85.6 \\
YRBC Annexin V+ & 100 & 0.1 \\
SeRBC Annexin V+ & 183 & 1.6 \\
\hline
\end{tabular}

Figure 4. Dot-plot of whole blood with the analysis strategy used to evaluate PS expression by binding to Annexin V+ in RBCs populations from different ages.

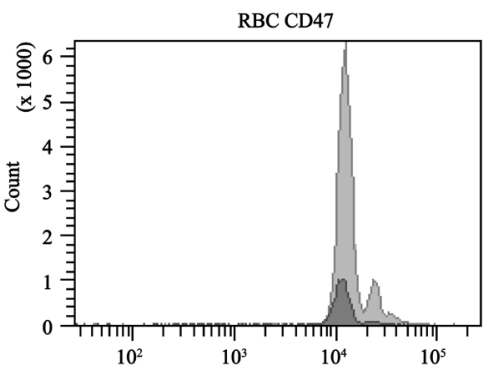

CD47 PE-A

\begin{tabular}{|lrrr|}
\hline Specimen Name: RBC CD47 & & & \\
& & & CD47 PE-A \\
Population & \#Events & \% Parent & Median \\
\hline$\square$ YRBC & 74,048 & 83.2 & 12,739 \\
$\square$ SeRBC & 12,700 & 14.3 & 11,571 \\
\hline
\end{tabular}

Figure 5. Histogram of CD47 expression in SeRBCs and YRBCs populations. 
Table 2. C3+ RBCs \% in SeRBCs and YRBCs populations.

\begin{tabular}{ccc}
\hline \multicolumn{2}{c}{ \% of RBCs C3+ } \\
\hline & SeRBCs & YRBCs \\
\hline Median \pm SD & $2.2 \pm 1.4$ & $0.2 \pm 0.6$ \\
\hline
\end{tabular}

Table 3. AV+ RBCs \% in populations of erythrocytes from different ages, evaluated through PS binding affinity to annexin $\mathrm{V}$.

\begin{tabular}{ccc}
\hline \multicolumn{2}{c}{$\%$ of RBCs Annexin V+ } \\
\hline SeRBCs & YRBCs \\
\hline Median \pm SD & $1.1 \pm 0.5$ & $0.15 \pm 0.1$ \\
\hline
\end{tabular}

Table 4. CD47 expression in RBCs populations from suspension 2.

\begin{tabular}{ccc}
\hline \multicolumn{2}{c}{ Median IF CD47 } \\
\hline SeRBCs & YRBCs \\
\hline Median \pm SD & $11010.0 \pm 882.9$ & $11965.0 \pm 945.7$ \\
\hline
\end{tabular}

The evaluation of erythrocyte senescence markers by flow cytometry was performed using information obtained from light scattering parameters and fluorescence intensity measurements. The forward scatter parameter corresponds to the scattered light collected between $0^{\circ}$ and $10^{\circ}$ and is related to the size of cells, while the side scatter value refers to the scattered light collected at $90^{\circ}$ and is related to the internal cells structure. Given that FSC represents cell size and SSC is related to the internal cellular complexity, when a sample of RBCs without prior separation step is acquired, we consider that the population of SeRBCs is in the area of lower FSC and greater dispersion of SSC, while the YRBCs fraction would be located in the region of higher FSC and more homogeneous SSC.

We have evaluated the usefulness of FC in the study of senescence erythrocyte, comparing the IgG percentages bound to SeRBCs and YRBCs fractions separated with Percoll and in samples without previous separation. The comparable values allowed us to investigate changes that take place during red blood cells aging, using a small amount of sample, avoiding separation method manipulations and decreasing the work time. In addition, the analysis strategy used in the results evaluation and the large number of cells studied allowed by FC, support this methodology election to continue our study of senescence markers in aged erythrocytes.

So far, numerous RBCs aging pathways have been proposed based on cellular changes identified in older RBCs. Band 3, a transmembrane protein that constitutes $25 \%$ of the total amount of RBC membrane proteins, has been postulated to be the major target of IgG isotype Nabs and might be a central step in senescent and damaged RBC clearance mediated by macrophages [24] [27] [28]. Among them, the clustering and/or breakdown of Band 3 is probably the central step in the major immunologically mediated pathway, leading to the generation of a powerful senescent signal, a senescent-specific neo-antigen. Similar proc- 
esses seem to be responsible for premature RBCs clearance in haemoglobinopathies, membrane protein deficiencies, Down's syndrome, Alzheimer's disease, etc [29] [30]. Free radical oxidation maybe an important factor underlying the formation of the senescent signal. There is evidence connecting the RBC oxidation levels in vivo to the breakdown of Band 3 and to the autologous IgG binding [31]. The senescent neo-antigen appearance induces the binding of both autologous IgGs and C3 complement fraction to the membrane, triggering erythrophagocytosis [32] [33]. NAbs activate classical complement pathway which yields a proportional number of deposited C3b [29].

In this study we observed that the C3b positive cells percentage is significantly different in the two regions: low FSC and high SSC (SeRBCs) over the area of the highest FSC and lowest SSC (YRBCs). These findings demonstrate a significant increase in C3b bound in SeRBCs populations. The C3b deposition indicates the participation of this complement component as an additional factor in the SeRBCs removal, making these cells more susceptible to erythrophagocytosis.

In healthy cells, phosphatidylserine is normally found on the inner leaflet RBCs membrane. However, in apoptosis a large increase of exposed PS amounts is seen. This increase of PS expression is proposed to be an "eat me" signal for an apoptotic cell phagocyte recognition, resulting in a non-inflammatory clearance of dying cells [34]. For a long time, it has been proposed that apoptotic cells that expressing PS can be cleared from circulation via macrophages by recognizing them through specific PS-receptors [8]. The analysis of PS expression in the erythrocyte membrane, evaluated by its annexin $\mathrm{V}$ binding affinity, showed an increased PS percentage in the region assigned to SeRBCs. This increase would allow its recognition by macrophages, contributing to their elimination. So, it could be considered as a signal that would lead to programmed cell death.

In erythrocytes, CD47 is part of the Rh complex and interacts with Band 3 and Protein 4.2 [35]. CD47 expression has been evaluated in a number of studies, but its role in RBCs human membrane has not been fully clarified. RBCs are cleared as a result of the accumulation of "eat me" signals on membrane via macrophage-dependent phagocytosis.

The conformational changes in CD47 expression serve as a switch from an erythrocyte phagocytosis inhibitory signal to a stimulating one. In CD47 expression FC investigation, we observed lower CD47 expression intensity in the region assigned to SeRBCs. Considering that CD47 is part of the Band 3 macro complex, the modifications in these proteins, mainly in Band 3, would have an indirect effect in CD47 conformation, triggering an inhibitory phagocytosis signal [36].

Further studies should be carried out by the proposed methodology to analyze other cell markers involved in the RBC senescence process.

\section{Conclusions}

During in vivo and in vitro aging, as well as in a variety of pathological conditions, RBCs display molecules that lead to recognition and removal of SeRBCs 
by immune system. Our results indicate that FC allows differentiation of RBCs populations with different sizes and densities. The aging markers studied allowed us to characterize cells of different ages. Therefore, this methodology could be an alternative tool to study erythrocyte aging using small sample quantities.

The identification of the process involved in RBCs aging constitutes a major challenge in current medicine research and transfusion. In this way, FC would contribute to the study of RBC progressive structural and biochemical alternations that occur during storage (RBC storage lesion). These findings would provide new insights into RBCs homeostasis.

\section{Ethics Approval and Consent to Participate}

The study protocol was accepted by the ethical committee of the School of Biochemistry Sciences of Rosario, Argentina and all participants provided written consent according to the principles of the Declaration of Helsinki.

\section{Authors' Contributions}

MAE participated in the design of the study, carried out the flow cytometry studies and drafted the manuscript. MLB participate in the experimental assay and helped to revise the manuscript. SGB helped to revise the manuscript. CC participated in the design of the study, performed the statistical analysis. CB conceived of the study, and participated in its design and coordination and helped to draft the manuscript. All authors read and approved the final manuscript.

\section{Conflicts of Interest}

The authors declare no conflicts of interest regarding the publication of this paper.

\section{References}

[1] Franco, R. (2012) Measurement of Red Cell Lifespan and Aging. Transfusion Medicine and Hemotherapy, 39, 302-307. https://doi.org/10.1159/000342232

[2] Cohen, R.M., Franco, R., Khera, P.K., Smith, E.P., Lindsell, C.J., Ciraolo, P.J., Palascak, M.B. and Joiner, C.H. (2008) Red Cell Life Span Heterogeneity in Hematologically Normal People Is Sufficient to Alter HbA1c. Blood, 112, 4284-4291. https://doi.org/10.1182/blood-2008-04-154112

[3] Mock, D.M., Matthews, N.I., Zhu, S., Strauss, R.G., Schmidt, R.L., Nalbant, D. Cress, G.A. and Widness, J.A. (2011) Red Blood Cell (RBC) Survival Determined in Humans Using RBCs Labeled at Multiple Biotin Densities. Transfusion, 51, 1047-1057. https://doi.org/10.1111/j.1537-2995.2010.02926.x

[4] Kay, M. (2005) Immunoregulation of Cellular Lifespan. Annals of the New York Academy of Sciences, 1057, 85-111. https://doi.org/10.1111/j.1537-2995.2010.02926.x

[5] Lutz, H.U. and Bogdanova, A. (2013) Mechanism Staging Senescent Red Blood Cells for Clearance in Healthy Humans. Frontiers in Physiology, 4, 387. https://doi.org/10.3389/fphys.2013.00387 
[6] Lutz, H.U. (2012) Naturally Occurring Anti-Band 3 Antibodies in Clearance of Senescent and Oxidatively Stressed Human Red Blood Cells. Transfusion Medicine and Hemotherapy, 39, 321-327. https://doi.org/10.1159/000342171

[7] Franco, R., et al. (2013) Changes in the Properties of Normal Human Red Blood Cells during in Vivo Aging. American Journal of Hematology, 88, 44-51. https://doi.org/10.1002/ajh.23344

[8] Nguyen, D.B., Wagner-Britz, L., Maia, S., Steffen, P., Wagner, C., Kaestner, L. and Bernhardt, I. (2011) Regulation of Phosphatidylserine Exposure in Red Blood Cells. Cellular Physiology and Biochemistry, 28, 847-856. https://doi.org/10.1159/000335798

[9] Zwaal, R.F., Comfurius, P. and Bevers, E.M. (2005) Surface Exposure of Phosphatidylserine in Pathological Cells. Cellular and Molecular Life Sciences, 62, 971-988. https://doi.org/10.1007/s00018-005-4527-3

[10] Lang, F., Gulbins, E., Lerche, H., Huber, S.M., Kempe, D.S. and Foller, M. (2008) Eryptosis, a Window to Systemic Disease. Cellular Physiology and Biochemistry, 22, 373-380. https://doi.org/10.1159/000185448

[11] Lang, F., Gulbins, E., Lang, P.A., Zappulla, D. and Foller, M. (2010) Ceramide in Suicidal Death of Erythrocytes. Cellular Physiology and Biochemistry, 26, 21-28. https://doi.org/10.1159/000315102

[12] Oldenborg, P.A., Zheleznyak, A., Fang, Y.F., Lagenaur, C.F., Gresham, H.D. and Lindberg, F.P. (2000) Role of CD47 as a Marker of Self on Red Blood Cells. Science, 288, 2051-2054. https://doi.org/10.1126/science.288.5473.2051

[13] Oldenborg, P.A., Gresham, H.D. and Lindberg, F.P. (2001) CD47-Signal Regulatory Protein Alpha (SIRPAlpha) Regulates Fcgamma and Complement Receptor-Mediated Phagocytosis. The Journal of Experimental Medicine, 193, 855-862. https://doi.org/10.1084/jem.193.7.855

[14] Barclay, A.N. and van den Berg, T.K. (2014) The Interaction between Signal Regulatory Protein Alpha (SIRPAlpha) and CD47: Structure, Function, and Therapeutic Target. Annual Review of Immunology, 32, 25-50.

[15] Burger, P., Hilarius-Stokman, P., de Korte, D., van den Berg, T.K. and van Bruggen, R. (2012) CD47 Functions as a Molecular Switch for Erythrocyte Phagocytosis. Blood, 119, 5512-5521. https://doi.org/10.1182/blood-2011-10-386805

[16] Piomelli, S. and Seaman, C. (1993) Mechanism of Red Blood Cell Aging: Relationship of Cell Density and Cell Age. American Journal of Hematology, 42, 46-52. https://doi.org/10.1002/ajh.2830420110

[17] Lutz, H.U., Stammler, P., Fasler, S., Ingold, M. and Fehr, J. (1992) Density Separation of Human Red Blood Cells on Self Forming Percoll R Gradients: Correlation with Cell Age. Biochimica et Biophysica Acta, 1116, 1-10. https://doi.org/10.1016/0304-4165(92)90120-J

[18] Steel, R.G.D., Torrie, J.H. and Dickey, D.A. (1997) Principles and Procedures of Statistics. A Biometrical Approach. 3rd Edition, McGraw Hill, New York.

[19] Kay, M.M. (1975) Mechanism of Removal of Senescent Cells by Human Macrophages in Situ. Proceedings of the National Academy of Sciences of the United States of America, 72, 3521-3525. https://doi.org/10.1073/pnas.72.9.3521

[20] Lutz, H.U. (2012) Naturally Occurring Anti-Band 3 Antibodies in Clearance of Senescent and Oxidatively Stressed Human Red Blood Cells. Transfusion Medicine and Hemotherapy, 39, 321-327. https://doi.org/10.1159/000342171

[21] Antonelou, M.H., Kriebardis, A.G. and Papassideri, I.S. (2010) Aging and Death 
Signalling in Mature Red Cells: From Basic Science to Transfusion Practice. Blood Transfusion, 8, s39-s47.

[22] Arashiki, N., Kimata, N., Manno, S., Mohandas, N. and Takakuwa, Y. (2013) Membrane Peroxidation and Methemoglobin Formation Are Both Necessary for Band 3 Clustering: Mechanistic Insights into Human Erythrocyte Senescence. Biochemistry, 52, 5760-5769. https://doi.org/10.1021/bi400405p

[23] Arese, P., Turrini, F. and Schwarzer, E. (2005) Band 3/Complement-Mediated Recognition and Removal of Normally Senescent and Pathological Human Erythrocytes. Cellular Physiology and Biochemistry, 16, 133-146. https://doi.org/10.1159/000089839

[24] Mohandas, N. and Gallagher, P.G. (2008) Red Cell Membrane: Past, Present, and Future. Blood, 112, 3939-3948. https://doi.org/10.1182/blood-2008-07-161166

[25] Tanner, M.J.A. (2002) Band 3 Anion Exchanger and Its Involvement in Erythrocyte and Kidney Disorders. Current Opinion in Hematology, 9, 133-139. https://doi.org/10.1097/00062752-200203000-00009

[26] Lutz, H.U. (2004) Innate Immune and Non-Immune Mediators of Erythrocyte Clearance. Cellular and Molecular Biology, 50, 107-116.

[27] Kay, M. (2005) Immunoregulation of Cellular Life Span. Annals of the New York Academy of Sciences, 1057, 85-111. https://doi.org/10.1196/annals.1356.005

[28] Hornig, R. and Lutz, H.U. (2000) Band 3 Protein Clustering on Human Erythrocytes Promotes Binding of Naturally Occurring Anti-Band 3 and Anti-Spectrin Antibodies. Experimental Gerontology, 35, 1025-1044. https://doi.org/10.1016/S0531-5565(00)00126-1

[29] Pantaleo, A., Giribaldi, G., Mannu, F., et al. (2008) Naturally Occurring Anti-Band 3 Antibodies and Red Blood Cell Removal under Physiological and Pathological Conditions. Autoimmunity Reviews, 7, 457-462. https://doi.org/10.1016/j.autrev.2008.03.017

[30] Kay, M.M., Bosman, G.J., Shapiro, S.S., et al. (1986) Oxidation as a Possible Mechanism of Cellular Aging: Vitamin E Deficiency Causes Premature Aging and IgG Binding to RBCs. Proceedings of the National Academy of Sciences, 83, 2463-2467. https://doi.org/10.1073/pnas.83.8.2463

[31] Kay, M.M., Goodman, S.R., Sorensen, K., et al. (1983) Senescent Cell Antigen Is Immunologically Related to Band 3. Proceedings of the National Academy of Sciences, 80, 1631-1635. https://doi.org/10.1073/pnas.80.6.1631

[32] Lutz, H.U., Stammler, P. and Fasler, S. (1993) Preferential Formation of C3b-IgG Complexes in Vitro and in Vivo from Nascent C3b and Naturally Occurring Anti Band 3 Antibodies. The Journal of Biological Chemistry, 268, 17418-17426.

[33] Boas, F.E., Forman, L. and Beutler, E. (1998) Phosphatidylserine Exposure and Red Cell Viability in Red Cell Aging and in Haemolytic Anemia. Proceedings of the National Academy of Sciences, 95, 3077-3081. https://doi.org/10.1073/pnas.95.6.3077

[34] Bruce, L.J., Beckmann, R., Ribeiro, M.L., Peters, L.L., Chasis, J.A., Delaunay, J., Mohandas, N., Anstee, D.J. and Tanner, M.J. (2003) A Band 3-Based Macrocomplex of Integral and Peripheral Proteins in the RBC Membrane. Blood, 101, 4180-4188. https://doi.org/10.1182/blood-2002-09-2824

[35] de Back, D.Z., Kostova, E.B., van Kraaij, M., van den Berg, T.K. and van Bruggen, R. (2014) Of Macrophages and Red Blood Cells: A Complex Love Story. Frontiers in Physiology, 5, 9. https://doi.org/10.3389/fphys.2014.00009

[36] Wiewiora, M., Piecuch, J., Sedek, L., Mazur, B. and Sosada, K. (2017) The Effects of Obesity on CD47 Expression in Erythrocytes. Cytometry Part B, 92, 485-491. https://doi.org/10.1002/cyto.b.21232 